\title{
On Foliations in Neighborhoods of Elliptic Curves
}

\author{
Mikhail B. Mishustin 1
}

Received: 13 April 2015 / Revised: 24 August 2015 / Accepted: 13 January 2016 /

Published online: 26 January 2016

(C) Institute for Mathematical Sciences (IMS), Stony Brook University, NY 2016

\begin{abstract}
A counterexample is given to a conjecture from the comments to Arnold's problem 1989-11 about the existence of a tangent foliation in a zero type neighborhood of an elliptic curve.
\end{abstract}

Keywords Neighborhood of elliptic curve $\cdot$ Foliation $\cdot$ Small denominator

\section{Introduction}

The comments to Arnold's problem 1989-11 [see Arnold (2004)] contain the following conjecture:

Let us have an elliptic curve with a zero self-intersection index in a complex surface. Then, in a neighborhood of this curve, there exists a foliation tangent to the curve.

Problem 1989-11 itself concerns holomorphic classification of neighborhoods of compact complex curves in complex surfaces. Its prehistory in the theory of dynamical systems is as follows.

Consider a saddle of a germ of an automorphism of $\mathbb{C}^{2}$. It has two separatrices. On any one of them, the automorphism can be reduced to a linear normal form. In the linearizing coordinate, it maps a small zero-centered circle to a circle of some other radius. These two circles bound an annulus. If we identify the circles using our automorphism, the annulus becomes an elliptic curve. If we make the same identification in a neighborhood of the annulus, this neighborhood becomes a neighborhood of this elliptic curve in some surface.

Mikhail B. Mishustin

mmishustin@ya.ru

1 Faculty of Mechanics and Mathematics, Moscow State University, Moscow, Russia 
Arnold (1976) suggested a construction to relate properties of the dynamical system to the properties of the complex structure of the neighborhood ${ }^{1}$. For example, holomorphic normal forms of such neighborhoods would imply holomorphic normal forms of such automorphisms, compact submanifolds of the neighborhood will become invariant submainfolds of the automorphisms, etc.

Following this idea, Arnold (1997, §27), studied two-dimensional neighborhoods of elliptic curves, having a zero self-intersection in these neighborhoods. Studying such neighborhoods was continued by Ilyashenko and Pyartli (1979), Ilyashenko (1982) and by the author of this note in Mishustin (1993, 1995).

Neighborhoods, studied by Arnold, appeared to be objects with "small denominators" or having "dense resonances" or belonging to "the Siegel case". This means that, for a dense subset of linear terms (the set of "resonant points"), geometry of objects radically changes, in particular, the dimensions of spaces of normal forms of finite jets of objects change. For such objects, the formal normalizing transformations may diverge. For non-resonant linear terms, smaller and smaller denominators appear in formal series which may cause divergence.

Small denominators appear in many mathematical models, starting with Poincare's normal forms of differential equations. A typical result for such models provides normal forms for "normally incommensurable" objects, whose linear terms are "not too well approximated by resonant ones" and delivers examples of divergence of formal normalizing transformations for other cases [see, for example, Bryuno (1971), Theorems II and III]. The resonant and abnormally commensurable cases are much harder to describe, despite lots of efforts. An exhaustive study of resonant germs of authomorphisms of $\mathbb{C}$ was performed by Voronin (1981). Bryuno (1971) constructed some partial normal forms for differential equations of this kind.

The linear term of the neighborhood is defined by the normal bundle of the curve in the surface. The normal bundle is resonant when some its symmetric power is holomorphically trivial. The normal bundle is normally incommensurable, when its $k$ th symmetric power is no closer to the trivial bundle than $C / k^{m}$ for suitable $C \in$ $\mathbb{R}^{+}, m \in \mathbb{N}$ (not depending on $k$ ). For this case, Ilyashenko and Pyartli (1979) proved that a neighborhood of the curve in a surface is biholomorphically equivalent to a neighborhood of the zero section in the space of the normal bundle.

The conjecture in question attempted to go a little further and to "decompose" a dynamic chaos to the "laminar" and "chaotic" components for any linear term. The hypothesis was based on the following two observations:

(a) If the normal bundle is normally incommensurable, then the foliation can be easily constructed in the space of the normal bundle. Let the normal bundle be abnormally commensurable. Include the neighborhood into a family of neighborhoods that deforms the normal bundle. In dense resonant members of the family, a covering curve will detach from the original curve. The detaching curve will also be elliptic, with a zero self-intersection and a zero intersection with the original curve. Its normal bundle will also be deformed by the family; therefore, in dense subset of the family, the covering of the detached curve will also detach, and so on. So, we

\footnotetext{
1 Instead of separatrices, Arnold used invariant submanifolds, the existence of which had been proved by Pyartli (1973) and Bryuno (1975).
} 
can expect rather a dense set of non-intersecting curves in a neighborhood of the original curve. We can suppose that they are compact leaves of some holomorphic foliation. The differentials of recurrence maps of this foliation along cycles on the original curve should be unitary, and this condition on foliation is necessary for non-linearizable neighborhoods.

(b) If we construct formal series of a foliation with unitary differentials of recurrence maps, it turns out that, for every small denominator, there will appear a small numerator conjugate to it. Thus, all finite jets of the foliation do not tend to infinity in resonant points, although they are not unique in resonances.

Arnold himself, though admitted this conjecture in the comment, treated it rather skeptically, because "it seemed unlikely that the neighborhoods could be constructed so easily". He was right.

\section{The counterexample}

Consider the trivial bundle over an elliptic curve supplied with coordinates $(r, \varphi)$ on fibers and the base respectively, $\varphi \sim \varphi+2 \pi \sim \varphi+\omega$. For the sake of definiteness, let $\omega=2 \pi i$.

Let $a(r, \varphi)$ be a function on a neighborhood of the zero section, smooth with respect to $\varphi$, holomorphic with respect to $r$, and vanishing for $r=0$. Let us define a nonstandard almost complex structure $J$ in this neighborhood given in the basis $(\partial / \partial r, \partial / \partial \varphi, \partial / \partial \bar{r}, \partial / \partial \bar{\varphi})$ by the matrix

$$
\left(\begin{array}{cccc}
i & 0 & 0 & -2 i a(r, \varphi) \\
0 & i & 0 & 0 \\
0 & 2 i \bar{a}(r, \varphi) & -i & 0 \\
0 & 0 & 0 & -i
\end{array}\right)
$$

The following facts are easily verified by a direct calculation:

(a) $J^{2}=-1$;

(b) the operator $\bar{\partial}_{J}=(d+J d J) / 2$ has a form of $\bar{\partial}_{J}=\bar{\partial}+d \bar{\varphi} \cdot a(r, \varphi) \cdot \partial / \partial r$;

(c) $\bar{\partial}_{J}^{2}=0$, because $a(r, \varphi)$ is holomorphic with respect to $r$.

The latter means that the structure $J$ is integrable, and the domain of $a(r, \varphi)$, equipped with the structure $J$, is a complex surface. Since $a(0, \varphi)=0$, the structure $J$ coincides with the standard structure on the zero section, hence the zero section is the same elliptic curve, embedded in this surface.

Suppose that, in a neighborhood of the curve, there exists a foliation, holomorphic with respect to the structure $J$ and tangent to the curve. In a smaller neighborhood of the curve, this foliation can be defined by the equation $d r=u(r, \varphi) d \varphi+v(r, \varphi) d \bar{\varphi}$ for suitable smooth $u(r, \varphi)$ and $v(r, \varphi)$.

The $J$-invariance of the foliation implies that $v(r, \varphi)=a(r, \varphi)$. 
Since the foliation is holomorphic on a two-dimensional complex manifold, it is integrable. The Frobenius condition implies:

$$
\begin{aligned}
\partial u / \partial \bar{r} & =0, \\
\partial u / \partial \bar{\varphi}-\partial v / \partial \varphi & =u \cdot \partial v / \partial r-v \cdot \partial u / \partial r .
\end{aligned}
$$

Equation (1) shows that $u(r, \varphi)$ is holomorphic with respect to $r$. Since the foliation is tangent to the curve, $u(0, \varphi)=0$. So we can expand $u$ and $v$ into series with respect to $r: u(r, \varphi)=\sum_{k \geq 0} u_{k}(\varphi) r^{k+1}$ and $v(r, \varphi)=\sum_{k \geq 0} v_{k}(\varphi) r^{k+1}$. Then (2) implies, for every $k \geq 0$,

$$
\partial u_{k} / \partial \bar{\varphi}=\partial v_{k} / \partial \varphi+\sum_{l+m=k}(m-l) u_{l} v_{m} .
$$

Let $a$ be of the following form: $a(r, \varphi)=r^{2}+r^{3}\left(e^{\varphi-\bar{\varphi}}+e^{i \varphi+i \bar{\varphi}}\right)$. Then $v_{1}(\varphi)=$ $1, v_{2}(\varphi)=e^{\varphi-\bar{\varphi}}+e^{i \varphi+i \bar{\varphi}}$, and $v_{k}=0$ for other $k$.

Expanding $u_{k}$ into Fourier series, $u_{k}(\varphi)=\sum_{l, m \in \mathbb{Z}} u_{k l m} e^{l(\varphi-\bar{\varphi})} e^{m(i \varphi+i \bar{\varphi})}$, we can successively solve Eq. (3) for $k=0,1,2,3 \ldots$

For $k=0$, we have $\partial u_{0} / \partial \bar{\varphi}=0$, so $u_{0}(\varphi)=u_{000}$.

For $k=1$, we have $\partial u_{1} / \partial \bar{\varphi}=u_{000}$. This equation can be solved only if $u_{000}=0$, and then $u_{1}(\varphi)=u_{100}$ and $u_{0}(\varphi)=0$.

For $k=2$, we have $\partial u_{2} / \partial \bar{\varphi}=\partial v_{2} / \partial \varphi=e^{\varphi-\bar{\varphi}}+i e^{i \varphi+i \bar{\varphi}}$, so $u_{2}(\varphi)=-e^{\varphi-\bar{\varphi}}+$ $e^{i \varphi+i \bar{\varphi}}+u_{200}$.

For $k=3$, we have $\partial u_{3} / \partial \bar{\varphi}=u_{1} v_{2}-u_{2} v_{1}=\left(u_{100}+1\right) e^{\varphi-\bar{\varphi}}+\left(u_{100}-1\right) e^{i \varphi+i \bar{\varphi}}-$ $u_{200}$. Solvability of this equation requires $u_{200}=0$, and then we have

$$
u_{3}(\varphi)=-\left(u_{100}+1\right) e^{\varphi-\bar{\varphi}}-i\left(u_{100}-1\right) e^{i \varphi+i \bar{\varphi}}+u_{300} .
$$

Let us continue this reasoning by induction for $k>3$, neglecting the terms of $e^{\varphi-\bar{\varphi}}, e^{i \varphi+i \bar{\varphi}}$ of degree $\geq 2$. We obtain the following induction step:

(a) By induction hypothesis,

$$
\begin{aligned}
u_{(k-2) 00}= & 0, \\
u_{k-1}(\varphi)= & (k-3) !\left[-\left(u_{100}+1\right) e^{\varphi-\bar{\varphi}}+i^{k-1}\left(u_{100}-1\right) e^{i \varphi+i \bar{\varphi}}\right]+u_{(k-1) 00} \\
& +(\text { terms of degree } \geq 2) .
\end{aligned}
$$

(b) Then

$$
\begin{aligned}
\partial u_{k} / \partial \bar{\varphi} & =-(k-2) u_{k-1}(\varphi) v_{1}-(k-4) u_{k-2}(\varphi) \cdot v_{2}(\varphi) \\
& =-(k-2) u_{k-1}(\varphi)+(\text { terms of degree } \geq 2) .
\end{aligned}
$$

Solvability of this equation requires $u_{(k-1) 00}=0$. Then

$$
\begin{aligned}
u_{k}(\varphi)= & (k-2) !\left[-\left(u_{100}+1\right) e^{\varphi-\bar{\varphi}}+i^{k}\left(u_{100}-1\right) e^{i \varphi+i \bar{\varphi}}\right]+u_{k 00} \\
& +(\text { terms of degree } \geq 2) .
\end{aligned}
$$


Now suppose that the series $\sum_{k \geq 0} u_{k}(\varphi) r^{k+1}$ converges in the closure of some domain $U_{\rho}:\{|r|<\rho\}$ for small enough $\rho>0$. In the space $L^{2}\left(U_{\rho}\right)$, corresponding to the standard metric $|d r|^{2}+|d \varphi|^{2}$, homogenous terms $u_{k}(\varphi) r^{k+1}$ are orthogonal to each other. Besides, in (4), the terms for $e^{\varphi-\bar{\varphi}}$, the terms for $e^{i \varphi+i \bar{\varphi}}$, and the sum of terms of degree $\geq 2$ of $e^{\varphi-\bar{\varphi}}$ and $e^{i \varphi+i \bar{\varphi}}$ are all orthogonal to each other. Thus,

$$
\|u\|_{L^{2}(U \rho)} \geq \sum_{k \geq 0}(k-2) ! \sqrt{\left|u_{100}+1\right|^{2}+\left|u_{100}-1\right|^{2}} \frac{(2 \pi)^{3 / 2} \rho^{k+2}}{\sqrt{2 k+4}} .
$$

Coefficients $\left(u_{100}+1\right)$ and $\left(u_{100}-1\right)$ cannot both be zero; so, in view of the factor $(k-2)$ !, the latter series diverges for any $\rho>0$.

Thus, for the trivial normal bundle, the counterexample has been constructed. It can be easily modified for any resonant bundle. The author believes that it is possible to adapt it to abnormally commensurable normal bundles.

\section{References}

Arnold, V.I.: Arnold's problems, 2nd edn. Springer-Verlag (2004)

Arnold, V.I.: Bifurcations of invariant manifolds of differential equations and normal forms in neighborhoods of elliptic curves. Funct. Anal. Appl. 10(4), 249-259 (1976). doi:10.1007/BF01076024

Arnold, V.I.: Geometrical methods in the theory of ordinary differential equations. Grundlehren der mathematischen Wissenschaften. Springer, New York (1997). http://books.google.ru/books? id=i-WJHniwLVwC

Bryuno, A.: The analytic form of differential equations. Tr. Mosk. Mat. O.-va 25, 119-262 (1971)

Bryuno, A.: Normal form of differential equations with a small parameter. Math. Notes 16, 832-836 (1975). doi:10.1007/BF01148130

Ilyashenko, Y.: Positive type imbeddings of elliptic curves into complex surfaces. Tr. Mosk. Mat. O.-va 45, 37-67 (1982)

Ilyashenko, Y., Pyartli, A.: Zero type neighbourhoods of embedded complex tori. Tr. Semin. Im. I. G. Petrovskogo 5, 85-95 (1979)

Mishustin, M.: Neighborhoods of Riemann curves in complex spaces. Funct. Anal. Appl. 29(1), 20-31 (1995) [translation from funkts. anal. prilozh. 29(1), 25-40 (1995)]. doi:10.1007/BF01077038

Mishustin, M.: Neighborhoods of the Riemann sphere in complex surfaces. Funct. Anal. Appl. 27(3), 1 (1993). doi:10.1007/BF01087535

Pyartli, A.: Birth of complex invariant manifolds close to a singular point of a parametrically dependent vector field. Funct. Anal. Appl. 6, 339-340 (1973). doi:10.1007/BF01077663

Voronin, S.: Analytic classification of germs of conformal mappings $(C, O) \rightarrow(C, O)$ with identity linear part. Funct. Anal. Appl. 15, 1-13 (1981). doi:10.1007/BF01082373 